\title{
Are Excipients Inert? Phenytoin Pharmaceutical Investigations with New Incompatibility Insights
}

\author{
Daniela Amaral Silva ${ }^{1,2}$, Neal M. Davies ${ }^{1}$, Raimar Löbenberg ${ }^{1}$ \\ ${ }^{1}$ Faculty of Pharmacy and Pharmaceutical Sciences, University of Alberta, $11361-87$ Avenue, Edmonton, AB. ${ }^{2}$ Faculty \\ of Pharmaceutical Sciences, University of Sao Paulo, 580 - Av. Prof. Lineu Prestes, - São Paulo - SP, Brazil.
}

Received, January 2, 2018; Revised, February 22, 2018; Accepted, March 28, 2018; Published, April 17, 2018.

\begin{abstract}
PURPOSE: The U.S. Pharmacopeia defines excipients as substances other than the active pharmaceutic ingredient (API) that are added in a drug delivery system in order to aid in the manufacturing process and enhance stability, bioavailability, safety, effectiveness and delivery of the drug. The 1968 phenytoin intoxication outbreak in Brisbane, Australia, is a classic example of an API-excipient interaction. When administered with $\mathrm{CaSO}_{4}$ the absorption of phenytoin was reduced due to an interaction between the API and the excipient. When $\mathrm{CaSO}_{4}$ was replaced by lactose, the amount of drug absorbed was much higher, resulting in the observed intoxication. It was hypothesized that phenytoin was converted to a calcium salt prior to ingestion. The purpose of this study was to mechanistically investigate the interactions between excipients and phenytoin to confirm the hypothesis of the previous reports. METHODS: Titration experiments with phenytoin and calcium salt were performed. Isothermal micro calorimetry was used to determine incompatibilities between excipients, phenytoin and milk. NMR was used to characterize the compounds. Dissolution tests containing $\mathrm{CaSO}_{4}$, lactose or sorbitol as excipients were also performed. Both Canadian and United States of America commercially available capsules were tested with milk and water. RESULTS: The calorimeter results indicate that phenytoin sodium interacts with $\mathrm{CaSO}_{4}$ in aqueous media and the dissolution profile of $\mathrm{CaSO}_{4}$ containing capsules showed a reduced dissolution rate. In addition, phenytoin sodium also interacts with lactose through a Maillard reaction that can occur at body temperature. Likewise, commercial Phenytoin sodium products interacted with milk and the products containing lactose showed browning in water. CONCLUSION: In Canada and the USA, the reference product contains lactose as an excipient in the formulation, whereas the Canadian generic formulations do not contain lactose. Any clinical relevance of these difference has not been determined. A new incompatibility between phenytoin and lactose has been discovered and an incompatibility with calcium was confirmed, which may have implications in regard to excipients and food effects.
\end{abstract}

This article is open to POST-PUBLICATION REVIEW. Registered readers (see "For Readers") may comment by clicking on ABSTRACT on the issue's contents page.

\section{INTRODUCTION}

The U.S. Pharmacopeia defines excipients as substances other than the active pharmaceutic ingredient (API) that are added in a drug delivery system in order to aid in the manufacturing process and enhance stability, bioavailability, safety, effectiveness and delivery of the drug. These substances have been appropriately evaluated for safety (1).

Although excipients are well characterized and evaluated for safety, they can interact with the API chemically or physically (2). An incompatibility can possibly affect the drug delivery performance and bioavailability, leading to loss of quality and potency, and compromising the safety and efficacy of the medication $(3,4)$. Thus, choosing the right excipients based on their function and compatibility with the API is of primary importance for a good quality drug product.

Phenytoin is an anti-epileptic drug related to the barbiturates in its chemical structure (5). Its therapeutic window ranges from 10 to $20 \mathrm{ug} / \mathrm{ml}$. This narrow safety margin makes therapeutic drug monitoring of utmost importance for this drug to maintain drug efficacy and safety (6).

The 1968 phenytoin intoxication outbreak in Brisbane, Australia, is a classic example of an API excipient interaction (7-9). According to Bochner et. al. (8) and the known evidence at the time, patients

Corresponding Author: Raimar Löbenberg, Faculty of Pharmacy and Pharmaceutical Sciences, University of Alberta, Edmonton, AB; E-Mail: raimar@ualberta.ca 
taking a certain brand of diphenylhydantoin (phenytoin) showed characteristic signs of intoxication related to the medication they were taking. After an extensive investigation the authors concluded that a change in excipient of the medication was the reason behind the changes in bioavailability and resultant intoxication. Studies (8) showed that when administered with $\mathrm{CaSO}_{4}$ as an excipient the absorption of phenytoin was jeopardized due to an interaction between the API and the calcium salt. When $\mathrm{CaSO}_{4}$ was replaced in the formulation by lactose, the amount of phenytoin absorbed was much higher, resulting in the observed intoxication.

Bochner et. al. (9) investigated capsules containing $\mathrm{CaSO}_{4}$ as an excipient taken by the patients and compared the solubility of the API in the formulation with that of phenytoin sodium. The study concluded that the solubility of phenytoin sodium had changed prior to ingestion and absorption was reduced. This finding alongside the recovery of phenytoin from the patient's feces confirmed their hypothesis of decreased alimentary absorption of phenytoin. The authors speculated that the formation of a calcium salt of phenytoin might be responsible for the much lower solubility.

The purpose of this study was to investigate further the interactions between excipients and phenytoin to mechanistically re-examine the hypothesis and interpretations of the previous studies.

\section{MATERIALS AND METHODS}

\section{MATERIALS}

Phenytoin USP grade and Calcium Sulfate NF were purchased from PCCA, USA (Houston, TX, USA; LOT: C172948 and LOT: C178073, respectively). Phenytoin sodium USP grade was obtained from Medisca ${ }^{\circledR}$ (Saint-Laurent, QC, Canada; LOT: 612840/A). Calcium chloride was purchased from Sigma-Aldrich. Lactose monohydrate was used from Meggle Wasserburg, Germany. Parteck ${ }^{\circledR}$ SI 150 (Sorbitol) was purchased from EMD Chemicals Inc. (Darmstadt, Germany; LOT: M285083). Regular milk and lactose free milk was purchased from a local grocery store and used before the expiration date. The powder mixture samples were put in glass ampoules and analyzed by thermal activity monitor III (TAM III) (TA instruments, USA). Commercial extended phenytoin sodium capsules were purchased: Dilantin (Pfizer Canada Inc, lot \#
T25924, exp. 05/2019), APO-Phenytoin Sodium (Apotex Inc. Toronto, Canada, lot \# NH9926, exp. 01/2019), Taro Pharmaceuticals U.S.A. Inc. (lot \# 316157 exp.03/01/19), Amneal Pharmaceuticals, U.S.A., LLC. (lot \# HL16617 exp.03/01/19).

Water for the dissolution tests and highperformance liquid chromatography (HPLC) analysis was purified by Elgastat Maxima UF and an Elgastat Option 3B water purifier by ELGA Laboratories Ltd. (Mississauga, ON, Canada) and then filtered. Gelatin capsules size 0 were used for the dissolution tests.

\section{METHODS}

\section{Titration}

A phenytoin solution was prepared by adding $1.4 \mathrm{~g}$ of phenytoin into a $250 \mathrm{~mL}$ of an alkaline $\mathrm{NaOH}$ solution. One portion of the phenytoin solution was titrated with calcium chloride $\left(\mathrm{CaCl}_{2}\right)$ in solution and the other portion with lactose solution. $\mathrm{H}^{1} \mathrm{NMR}$ was performed to analyze the precipitate obtained in the titration with the $\mathrm{CaCl}_{2}$ solution.

\section{Calorimeters experiments}

Samples were analyzed by thermal activity monitor III (TAM III) (TA instruments, USA) to investigate excipient-API interactions, e.g. as solid-state reactions or in solution. The microcalorimeter ampoule experiment was selected and the experiments were performed at $40^{\circ} \mathrm{C}$.

Two scenarios were investigated: dry powder mixtures and water $(1 \mathrm{ml})$ added to the powders mixtures, as described in Table 1. After adding the given compound combination into the ampoules, they were vortexed yielding a homogenous mixture, and then put into measuring position in the calorimeter. The experiments ran for a minimum of three days and ended after a flat line was obtained.

Furthermore, commercially available capsules from Canada and the United States of America were tested. The content of the capsules obtained from the market was transferred into the calorimeter vials with milk, water or lactose free milk as solvents.

\section{Dissolution tests}

Capsules were prepared by adding the powder mixture (API and excipients) one by one using a 2:1 ratio between excipient and API. The excipients used were lactose, $\mathrm{CaSO}_{4}$ or sorbitol. For the dissolution tests a VK 7020 system (Varian Inc.) coupled with VK 8000 auto sampler (Varian Inc.) was used. 
Table 1. Powder mixtures composition used in the calorimeter experiments

\begin{tabular}{cc}
\hline Dry powder mixtures & Powder mixtures with water \\
\hline Phenytoin sodium & Phenytoin sodium \\
Calcium Sulfate & Calcium Sulfate \\
Phenytoin sodium & Phenytoin sodium \\
Lactose & Lactose \\
Phenytoin & Phenytoin \\
Magnesium Sulfate & Lactose \\
Phenytoin & Lactose* \\
Sodium Sulfate & Phenytoin* \\
Phenytoin & Phenytoin sodium* \\
Calcium Sulfate & Water* \\
\hline Phenytoin sodium* & Calcium Sulfate* \\
Phenytoin* & \\
\hline Calcium Sulfate* & \\
Lactose* & \\
\hline Magnesium Sulfate* & \\
Sodium Sulfate* & \\
\hline *Controls - Mixtures of milk with both phenytoin sodium and phenytoin were also analyzed. \\
\hline
\end{tabular}

All dissolution tests were performed according to the USP monograph "prompt phenytoin sodium capsules" (10) with additional sample points. In brief: USP Apparatus 1, $900 \mathrm{~mL}$ dissolution media (water), $50 \mathrm{rpm}$ rotation speed and temperature set at $37.0^{\circ} \mathrm{C}$. Samples were collected at 5, 10, 15, 20, 30 minutes and quantified in a VP-class Shimadzu Scientific Instruments (Kyoto, Japan) liquid chromatograph, equipped with a Lichrospher ${ }^{\circledR} 60$ RP Select B column $(5 \mu \mathrm{m}, 12.5 \times 4 \mathrm{~mm})$.

\section{STATISTICAL ANALYSIS}

The Microsoft Excel ${ }^{\mathrm{TM}}$ add-in DDSolver was used to analyze the dissolution data. The dissolution profiles for the lactose and $\mathrm{CaSO}_{4}$ containing formulations were compared by $\mathrm{f} 2$ statistics. The factor $\mathrm{f} 2$ is a similarity factor that measures the closeness between two profiles (11). According to the FDA criteria, f2 value between $50-100$ indicates similarity between two dissolution profiles (12).

\section{RESULTS}

\section{Titration}

The titration experiments with $\mathrm{CaCl}_{2}$ and lactose were performed to verify the precipitation of phenytoin. A precipitate was only obtained when performing the titration with the calcium salt, confirming that, in solution, phenytoin interacted with calcium forming a product compound with low solubility $(9,13,14)$. Figure 1 shows $\mathrm{H}^{1} \mathrm{NMR}$ result of the precipitate with calcium chloride, confirming that the precipitate was phenytoin.

\section{Calorimeter experiments}

Experiments performed using powder mixtures are shown in Figure 2. Since no heat flow was observed a solid-state reaction as reported by Bochner et. al. (9) could not be confirmed neither for phenytoin sodium nor for phenytoin.

Considering the above, another experiment was conducted with water added to all powder mixtures (Figure 3). Phenytoin sodium interacts with calcium sulfate (red line) in the presence of water, and also with lactose (blue line). 


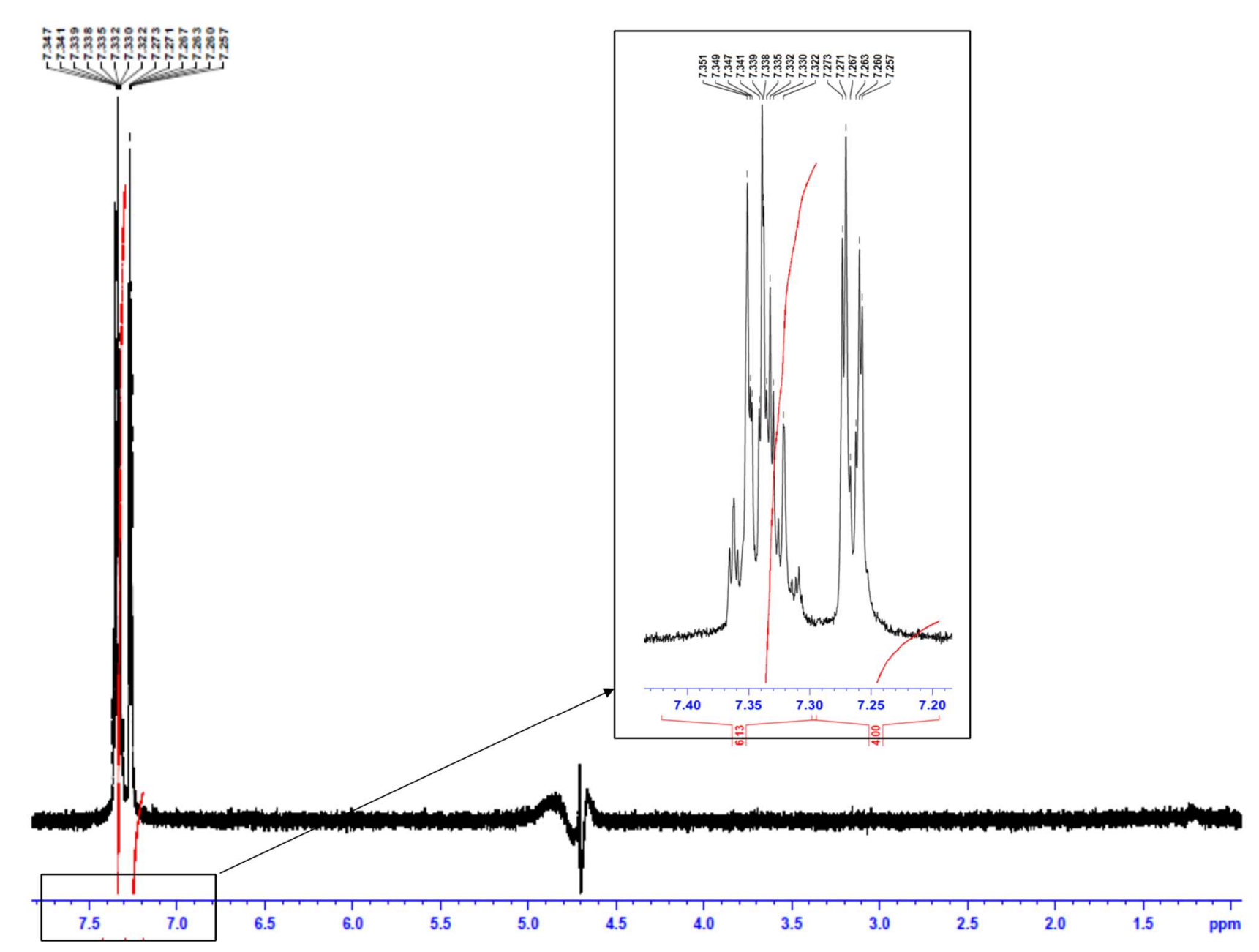

Figure 1. $\mathrm{H}^{1} \mathrm{NMR}$ spectrum for the precipitate obtained in the titration of phenytoin solution with $\mathrm{CaCl}_{2}$. When lactose was used as an excipient no precipitation was observed, nevertheless, after some time, the titrated solution became a yellow colour.

The ampoule containing phenytoin sodium, calcium sulfate and water mixture formed crystals on the wall of the vial. Hence, both the heat flow (red line in Figure 3) and the crystals formed suggest that phenytoin sodium interacts with $\mathrm{CaSO}_{4}$ in aqueous medium.

The increased heat flow in Figure 3 (blue line) corresponds to the mixture of phenytoin sodium, lactose and water. After experimental completion, the inside of the ampoule demonstrated the presence of a brown compound formed, suggesting a Maillard reaction in aqueous medium between phenytoin sodium and lactose. The heat flow (blue line in Figure 3) and the brown compound formed suggest that phenytoin sodium not only is incompatible with $\mathrm{CaSO}_{4}$ and precipitates, but it is also incompatible with lactose and reacts. This was further confirmed and verified by HPLC. No phenytoin peak was detected (data not shown) when the brown compound was analyzed, suggesting that phenytoin sodium had been converted into another compound.

However, no reaction between phenytoin as free form and lactose was observed in aqueous media. This shows differential effects of a salt and the free form interacting with an excipient. This was further confirmed by the flat line (orange line) in the microcalorimeter experiment in Figure 3. The negative heat flow in the first 22 hours observed for the orange and green lines is suggested to be due to the solubilization of lactose in water.

To further investigate this finding, a calorimeter experiment was performed with mixtures of milk with both phenytoin and sodium phenytoin, as shown in. Figure 4. Upon completion of the experiment, the vial with the phenytoin sodium and milk mixture yielded a yellow colour, whereas the mixture with the free base and milk did not. 


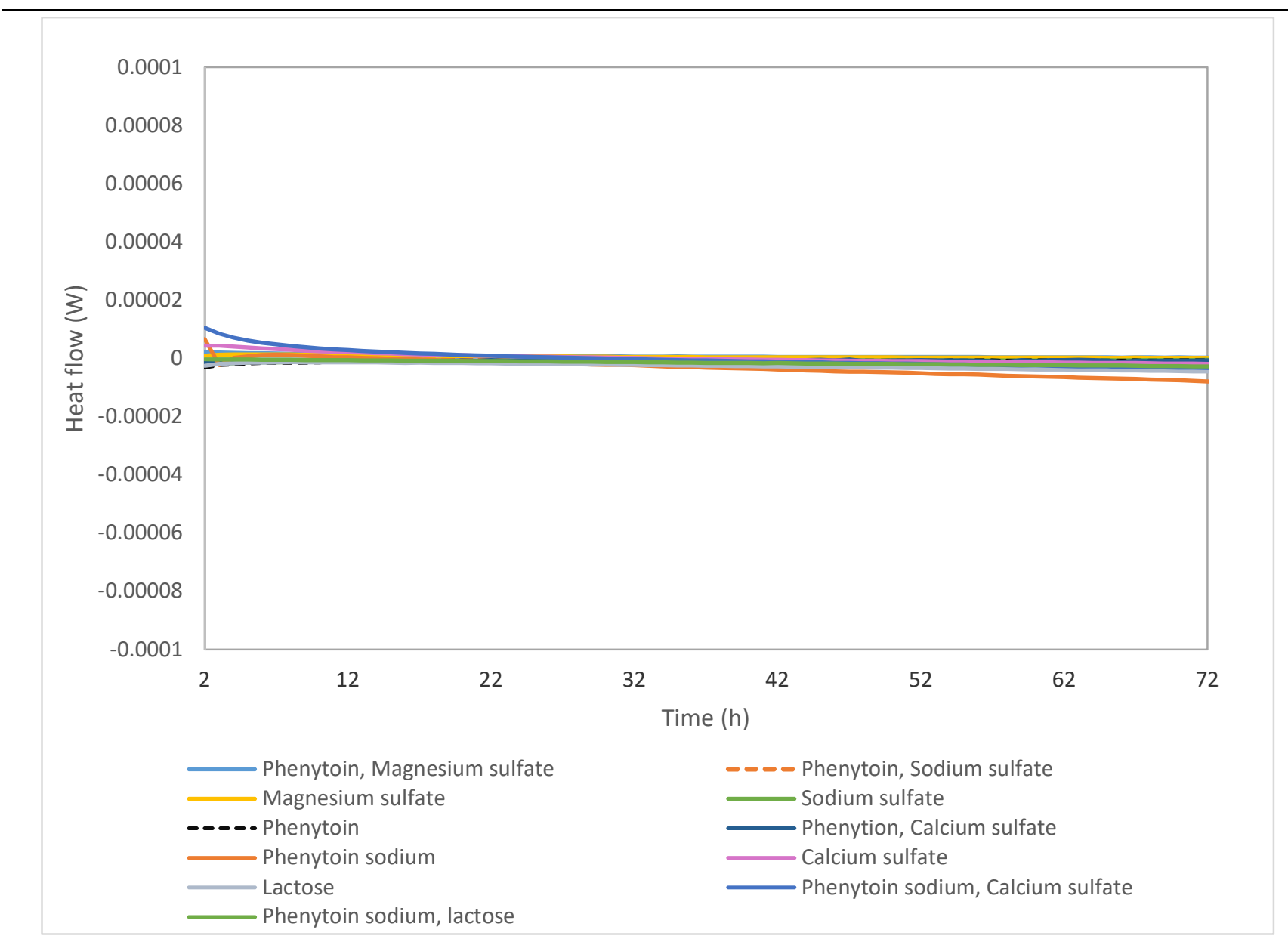

Figure 2. Calorimeter experiments result for the dry powder mixtures.

Finally, commercially available products were tested with milk to assess whether such a reaction would take place (Figure 5). The heat flow indicated that all tested products interacted with milk. This was confirmed by the Maillard reaction which turned the samples brown.

For comparison purposes the above-mentioned formulations were also tested in water and lactose free milk (Figure 6). In water, only lactose containing formulations (Dilantin - Pfizer Canada Inc. and Taro Pharmaceutics U.S.A.) resulted in browning. In lactose free milk a slight tinge of yellow colour was obtained for the non-containing lactose formulations due to traces of lactose in the product.

\section{Dissolution}

Dissolution tests using USP apparatus 1, $50 \mathrm{rpm}$ and water $900 \mathrm{~mL}$ are the USP recommended parameters for "prompt release phenytoin sodium capsules" (6), this monograph was recently withdrawn from the
USP (15) because this dosage form is not used for human use in the United States. However, the capsules prepared by adding the powder mixture (API and excipients) one by one using a 2:1 ratio between excipient and API showed a suitable dissolution profile using this method (Figure 7) rather than the official USP method which is for extended release capsules.

As shown in Figure 7 and according to the $\mathrm{f} 2$ test performed (f2: 52) for the lactose and $\mathrm{CaSO}_{4}$ containing formulations the dissolution profiles were similar when using water as the dissolution medium. Since $<85 \%$ release in 15 minutes was obtained for both profiles, they were compared and found as similar (f2 test $=52$ ).

The $\mathrm{f} 2$ test was not performed for the sorbitol containing formulation because $>85 \%$ release was obtained in 15 minutes, demonstrating that the release profile for this formulation is not similar to the other two. Sorbitol was chosen as an excipient because it is not a reducing sugar and it does not interact with phenytoin. 


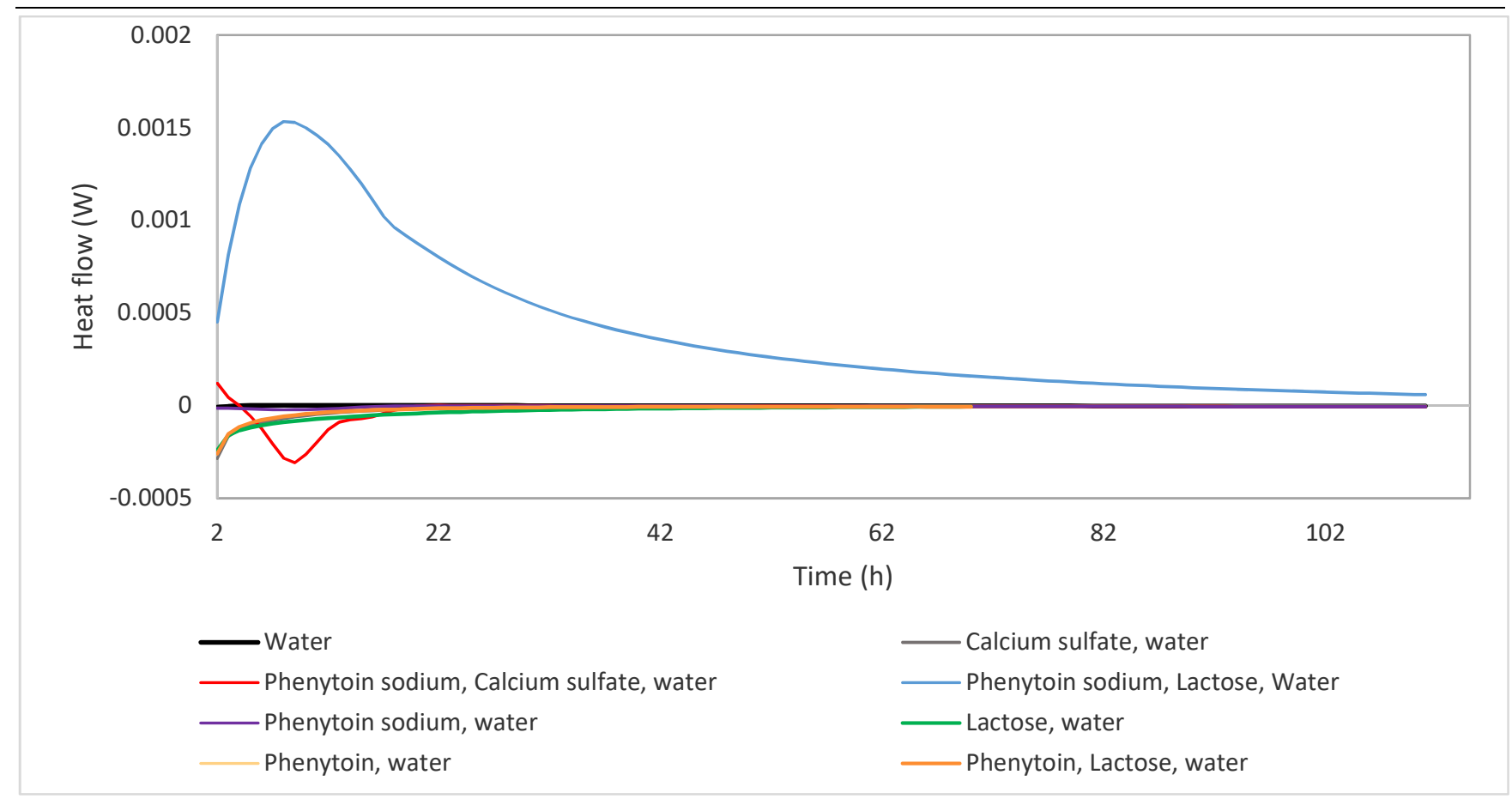

Figure 3. Calorimeter experiments result for the powder mixtures with water added.

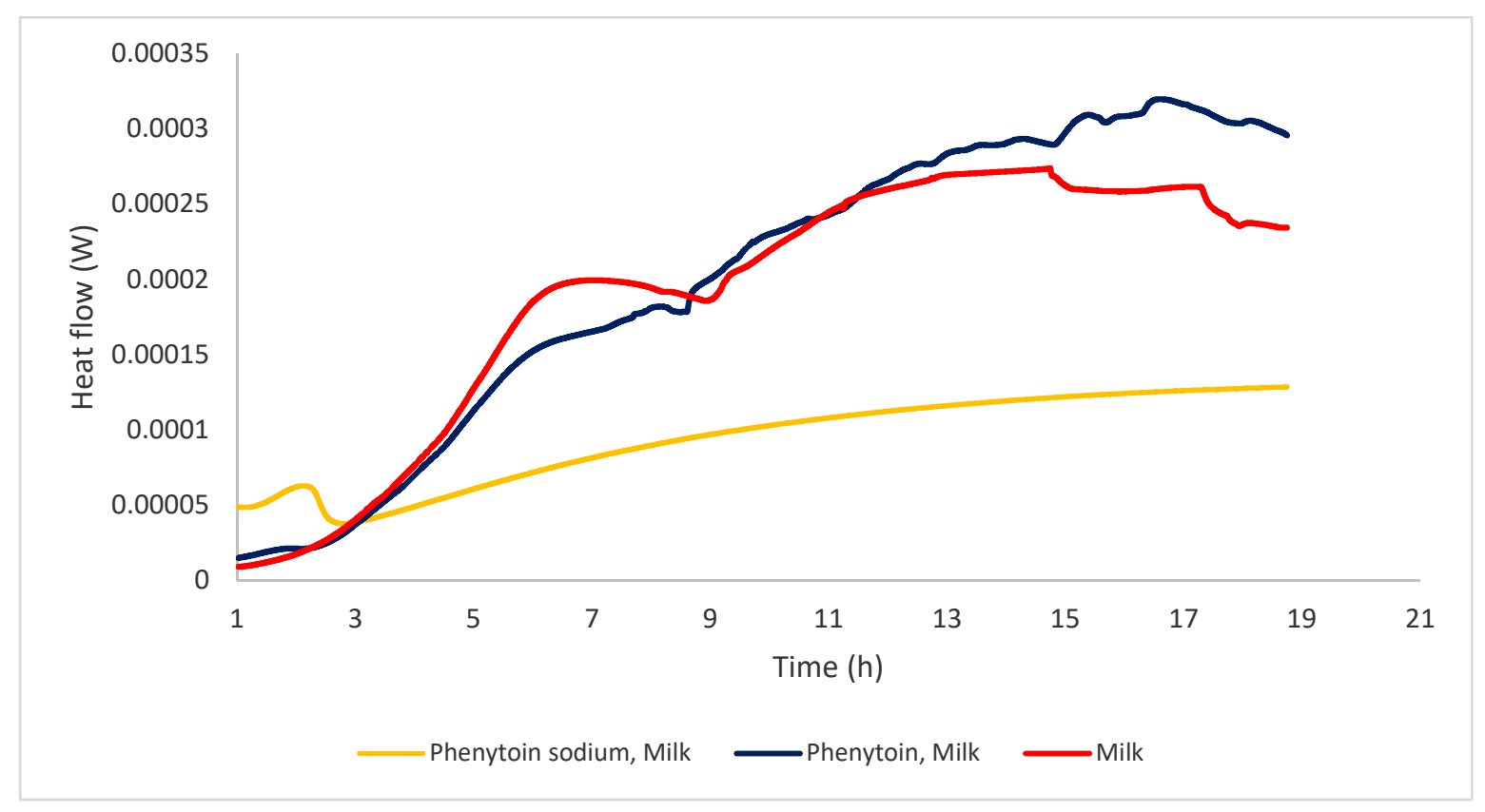

Figure 4. Calorimeter experiments result for the mixtures: milk and phenytoin; milk and sodium phenytoin.

The formulation containing sorbitol had a higher and faster release rate than the other formulations. At 30 minutes the amount released was statistically different for the sorbitol containing formulation compared to the lactose containing formulation and similar to the formulation with $\mathrm{CaSO}_{4}$. Our dissolution results demonstrate that the rate but not the extent of dissolution and not solubility was affected.

In addition, the capsules containing lactose were slightly yellow (Figure 8) at the end of the dissolution test, implying that a Maillard reaction was occurring at body temperature. 


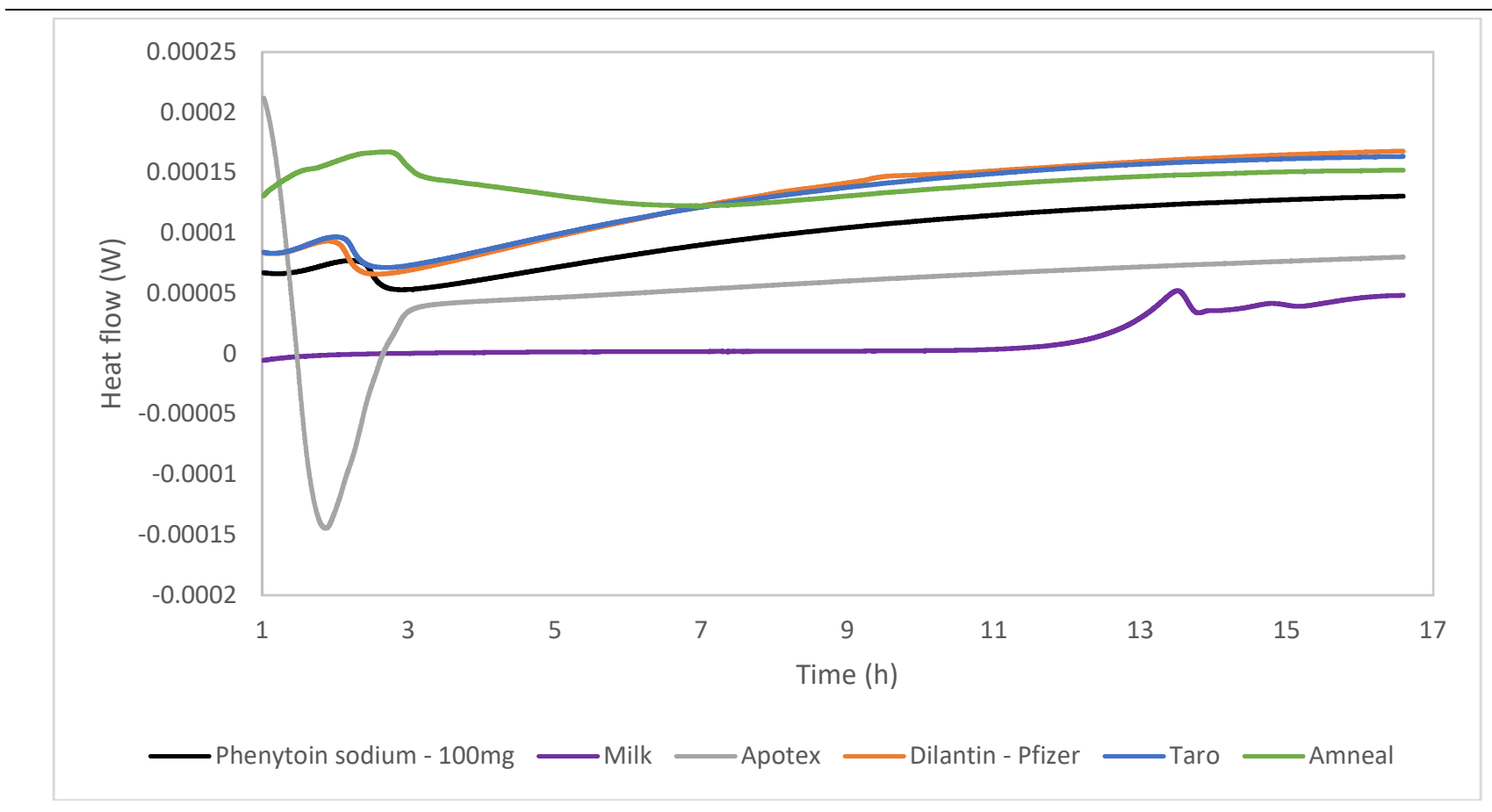

Figure 5. Calorimeter experiments result for commercially available extended release phenytoin sodium capsules (100 mg) in milk.

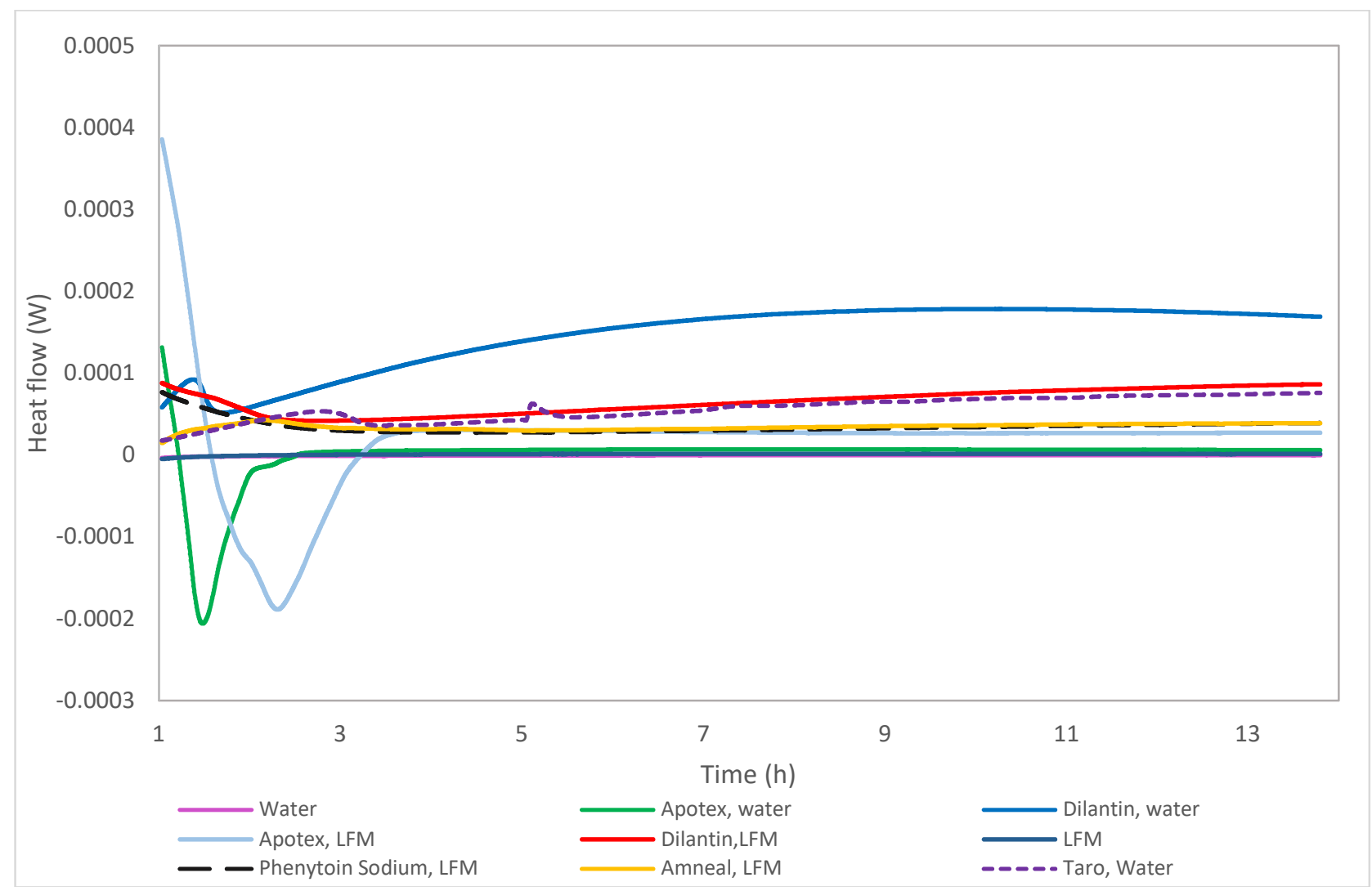

Figure 6. Calorimeter experiments result for commercially available extended release phenytoin sodium capsules (100 mg) in water and lactose free milk (LFM). 


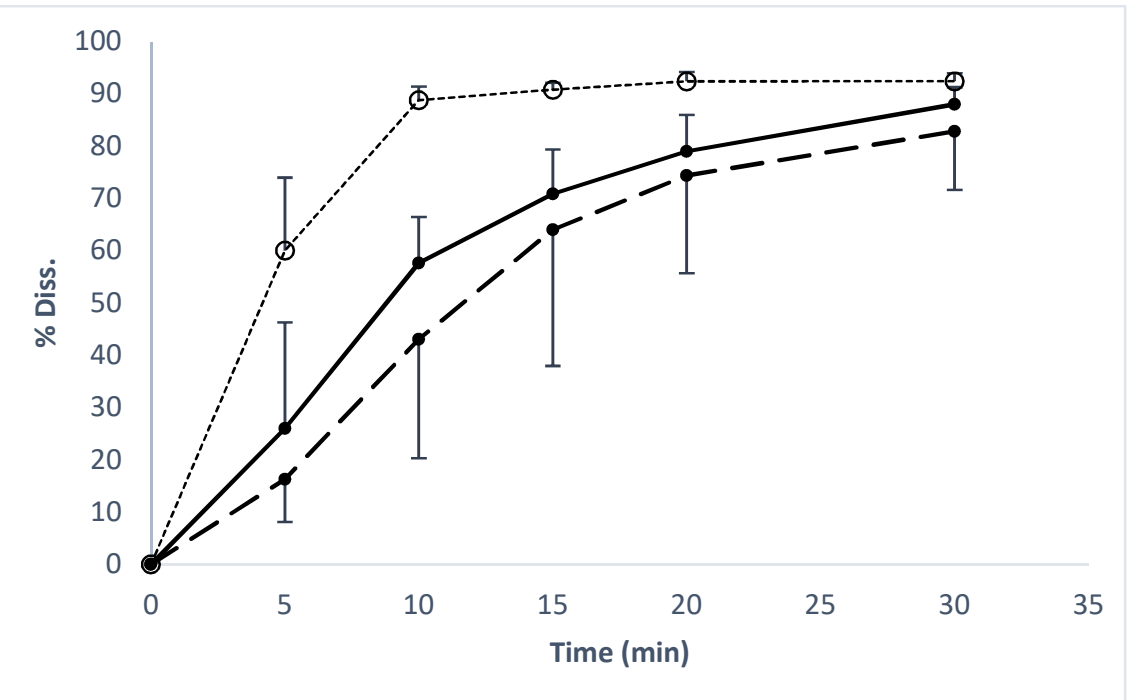

Figure 7. Dissolution profiles obtained for phenytoin sodium with lactose (dashed line), phenytoin sodium with $\mathrm{CaSO}_{4}$ (solid line) and phenytoin with sorbitol (dotted line and open circles) capsules in water.

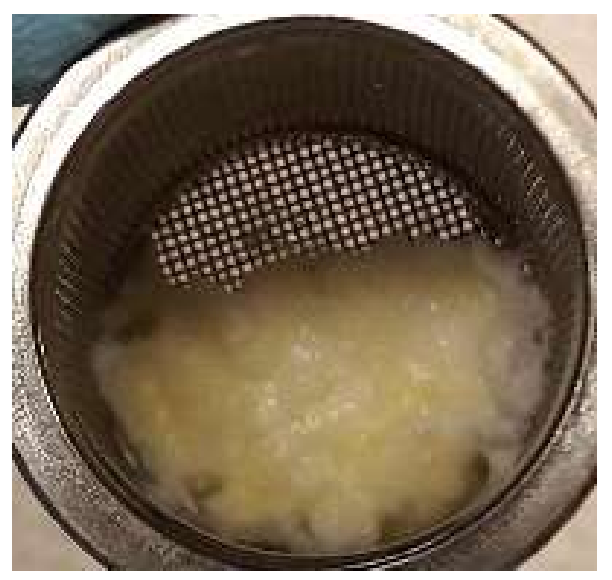

Figure 8. Phenytoin sodium and lactose capsules after the dissolution tests. After 30 minutes at body temperature $\left(37.0^{\circ} \mathrm{C}\right)$ the capsules became yellow.

\section{DISCUSSION}

In 1972 after studying the phenytoin excipient effect in patients Bochner et al (9) concluded that $\mathrm{CaSO}_{4}$ was the excipient component in the formulation that interacted with phenytoin sodium yielding a compound with different solubility than phenytoin sodium itself. They reported that, prior to ingestion, the capsules containing $\mathrm{CaSO}_{4}$ had almost $25 \%$ of phenytoin with altered solubility properties, however, the mechanism by which the conversion occurred was not experimentally delineated.

As shown in Figure 2, our data cannot confirm a solid-state reaction between $\mathrm{CaSO}_{4}$ and phenytoin sodium. Our results demonstrated that a reaction occurs (red line in Figure 3) in the presence of water. Thus, it is suggested that the interaction between phenytoin sodium and $\mathrm{CaSO}_{4}$ could occur in situ or in a manufacturing process where humidity is used, such as wet granulation. However, we know from discussions with a manufacturer that humidity in the manufacturing process is strictly controlled due to the hygroscopic properties of the API. (Dr. J. Cook, personal communication, November 28, 2017).

A possible explanation for the reduced solubility of phenytoin sodium prior to ingestion in the capsules containing $\mathrm{CaSO}_{4}$ is the absorption of moisture and $\mathrm{CO}_{2}$ from the atmosphere providing the needed conditions for the API-excipient interaction to occur (16). Newton DW et. al. (16) has already 
reported that in bottles of phenytoin sodium powder the solubility decreased over time due to absorption of moisture and transformation of phenytoin sodium to the free form.

In the dissolution experiments (Figure 7) it was observed a slower dissolution rate but not a lower solubility for the $\mathrm{CaSO}_{4}$ capsules we formulated. However, phenytoin precipitated (Figure 1) when titrated with a calcium salt solution. The effect of convection in a dissolution experiment may limit crystal formation. In addition, in the dissolution vessel a medium volume of $900 \mathrm{~mL}$ in which both the phenytoin sodium and calcium salt solubilize is much larger than the titration studies with a smaller volume of $100 \mathrm{~mL}$ where more concentrated solutions of phenytoin and calcium are present.

Since the 1968 phenytoin intoxication outbreak in Australia much attention was drawn to the incompatibility between phenytoin and $\mathrm{CaSO}_{4}(17-$ 20). However, our results show that phenytoin sodium is also not compatible with lactose. This incompatibility was evident due to the colouring after both dissolution and calorimeter experiments. The yellow-brown colour suggests that a Maillard reaction is occurring between the two compounds, given that phenytoin sodium has a nitrogen with a negative charge and lactose is a reducing sugar (4). On the other hand, phenytoin acid did not react with lactose in the same way that the sodium salt did (Figure 3). This may be due to the lack of the negative charge on the nitrogen in the free form.

The presence of an amine and a reducing sugar alone may not be enough for the Maillard reaction to happen, other factors besides ionization seem to play a role too. For example, lactose concentration in the product and the solid-state form of lactose can also impact API-excipient interactions since its crystalline form is considered to be less reactive than the amorphous one $(21,22)$. In addition, other factors such as moisture and temperature can also play also an important role in a Maillard reaction.

The incompatibility between phenytoin sodium and lactose could represent a loss of drug when patients take such formulation since this reaction can occur at body temperature (Figure 8).

Continuing with the investigation on how this reaction would take place, phenytoin sodium was mixed with milk and analyzed through microcalorimetry. The heat flow of milk only and phenytoin/milk mixture were similar while the phenytoin sodium/milk mixture demonstrated a reduced heat flow pattern. The vials containing milk and phenytoin with milk did not show any difference in colour. The blue and red lines in Figure. 4 could represent bacteria growth, since the pattern is similar to the ones obtained in other milk calorimetry studies $(23,24)$. Maillard reactions are exothermic (25-27) and this explains the higher heat flow in the first two hours in the phenytoin sodium/milk sample mixture (yellow line in Figure 4). The lactose readily reacted with phenytoin sodium and the heat flow is taking place at a lower rate (yellow line Fig. 4 from 3 hours on) due to the decreased amount of lactose.

Furthermore, the calorimetry experiments performed with commercial products showed that phenytoin sodium interacted with milk regardless of the formulation composition (Figure 5). When tested in water, only the lactose containing products showed browning, confirming, once again, the incompatibility between API and excipient.

According to the Canadian Food Inspection Agency "lactose- free means that there is no detectable lactose in the food using an acceptable analytical method". Therefore, lactose free milk can have some acceptable remaining amount of lactose. This explains the slight colouration observed for the Apotex and Amneal products with lactose free milk. The more evident browning for Dilantin in lactose free milk may be due to interaction with the additional lactose in the formulation, as colour change was observed in water. The negative heat flow for the Apotex product can be attributed to the disintegration process of the minitablets. The colour change intensity for each formulation in the different tested media is summarized in Table 2.

In previous studies by Macheras et al. the authors investigated the effect of milk on the solubility of phenytoin sodium (28). They report a significant increase in solubility, but surprisingly no incompatibility between the API and milk is reported. However, in an in vivo study administering $200 \mathrm{mg}$ phenytoin sodium capsules with water or milk a significant decrease in AUC and Cmax were observed when milk was used. The AUC dropped from $151.2 \pm 72.2 \mu \mathrm{g} \mathrm{h} \mathrm{mL}^{-1}$ (control with water) to $81.9 \mu \mathrm{g} \mathrm{h} \mathrm{ml}^{-1}( \pm 21.3)$ (control with milk) and Cmax dropped from $3.3( \pm 0.7) \mu \mathrm{g} \mathrm{ml}-1$ to $2.6( \pm 0.5) \mu \mathrm{g} \mathrm{ml}{ }^{-}$ 1. This indicates a food effect with milk for the tested formulations due likely to the presence of both and lactose and calcium.

In another study by Neuvonen et al. (29) patients received phenytoin as a free acid with milk or water. No differences in Cmax were observed. The authors concluded that "a direct physicochemical interaction between phenytoin and milk constituents is unlikely" since the absorption of phenytoin was not changed. 
This is consistent with and corroborates our in vitro results regarding the lack of interaction between phenytoin free acid and lactose.

The change in excipients (from $\mathrm{CaSO}_{4}$ to lactose) by Parke-Davis was undertaken in the early 1960's $(8,9)$ and ever since the reference product Dilantin (Pfizer) contains lactose as an excipient $(5,8,30)$. Its monograph in Canada was last revised in August, 2017 (30). Interestingly, in Canada, the generic brands Apotex Canada Inc. and Taro Pharmaceuticals Inc. of extended phenytoin sodium capsule do not contain lactose as an excipient $(31,32)$. It is noteworthy that the formulations with and without lactose were bioequivalent, which is different than the food effect observed with milk. The bioequivalence results might be due to the slow release of the drug from the formulation. The USP requires an in vitro release of 45,65 and $70 \%$ at 30 , 60, $120 \mathrm{~min}$ (33). In vivo the lactose and the API might dissolve at different rates.

Also, the innovator product was bioequivalence tested in a fasted / fed study (34) with milk as one of the components of the meal. No bioequivalence problems were observed. This might be due to the dilution of the milk throughout the entire meal, which could be at variance with the study (28) where only milk was utilized. In addition, according to a query to the Canada Vigilance Program no reports of lactose or milk interactions with phenytoin were found from 1965 - 2017 (35).

Moreover, the other marketed products in the USA also don't contain lactose as an excipient (3639), with exception of Taro Pharmaceuticals (40), which is intriguing, as their approved product in Canada does not contain lactose in its formulation. In a food effect study comparing the innovator product to a particular generic product, it was found that the generic formulation was within the bioequivalence criteria of the FDA. However, the authors state: "when taking phenytoin sodium with food, product switches may result in either side effects or loss of seizure control" (41). How much of this (if any) can be attributed to excipient API effects or the API dissolution is not known.

The published literature appears to be contradictory and the amount of lactose necessary to cause an interaction that results in a change in bioavailability remains unanswered. The in vitro tests may be overly discriminatory for the in vivo performance. Our in vitro results suggest that amounts of dissolved API and lactose at the same location within the gastrointestinal tract may facilitate a Maillard reaction. Nevertheless, the lactose amount needed for the reaction to happen in vivo affecting the drug's bioavailability is unknown, once it is a different milieu than the in vitro conditions of a dissolution vessel.

Hence, considering the excipient concentrations in approved phenytoin products, it becomes clear that even with the most commonly used excipients, which are generally considered "pharmaceutically inert", a chemical or physical interaction with the active pharmaceutical ingredient could compromise the in vitro dissolution testing and potentially alter bioavailability and bioequivalence. Bioequivalence and bioavailability tests can delineate the effect of potential interactions, an awareness of the issue at the development level could save time and cost. However, despite evidence of this in vitro interaction and potential interaction in vivo there does not yet appear to be any definitive reduction of bioavailability for contemporary commercial Canadian and USA brands that contain lactose leading to bioinequivalence issues nor, to the best of our knowledge, have any therapeutic failures due to the interaction of phenytoin products with milk/lactose has been reported in Canada.

\section{CONCLUSION}

Our study mechanistically investigated previous reports of excipient-API interactions with phenytoin. The calorimeter experiments results indicate that phenytoin sodium interacts with $\mathrm{CaSO}_{4}$ in aqueous media. Furthermore, phenytoin sodium also interacts with lactose through a Maillard reaction at body temperature which could possibly lead to bioavailability variations if administered with lactose containing milk.

In Canada and the USA, the reference product still has lactose as an excipient in the formulation listed since it was changed in the 1960's, whereas all Canadian and most USA generic formulations do not contain lactose.

The current bioequivalence data do not suggest that a potential lactose-phenytoin sodium interaction challenges the therapeutic equivalence between the available products. However, assuming that the commonly used excipients are so called inert could still cause potential development issues.

Phenytoin was first introduced as drug product in 1938 and much has been learned about drugexcipient incompatibility with calcium, but even after of eighty years of clinical use a new incompatibility between phenytoin and lactose has been experimentally delineated in vitro. 
Table 2. Excipients composition of different approved products of extended release phenytoin sodium capsules $100 \mathrm{mg}$ in Canada and in the USA, and colour change intensity for the tested products in Milk, lactose free milk (LFM) and water

\section{Company}

\section{Excipients composition} Canada

Parke-Davis (Pfizer)* Reference

\begin{tabular}{|c|c|}
\hline Apotex* & $\begin{array}{l}\text { Colloidal silicon di } \\
\text { and magnesium ste }\end{array}$ \\
\hline Taro Pharmaceuticals & $\begin{array}{l}\text { Lactitol monohydr } \\
\text { sulphate, and talc. }\end{array}$ \\
\hline $\begin{array}{l}\text { Parke-Davis (Pfizer) } \\
\text { Reference }\end{array}$ & Lactose monohy \\
\hline Taro Pharmaceuticals* & $\begin{array}{l}\text { Lactose monohy } \\
\text { hypromellose. }\end{array}$ \\
\hline Mylan Pharmaceuticals Inc. & $\begin{array}{l}\text { Colloidal silicon d } \\
\text { magnesium oxide, } \\
\text { cellulose, povidon }\end{array}$ \\
\hline Sun Pharmaceutical Industries, Inc. & $\begin{array}{l}\text { Lactitol monohy } \\
\text { magnesium stear }\end{array}$ \\
\hline Amneal Pharmaceuticals* & $\begin{array}{l}\text { Hydroxypropyl ce } \\
\text { talc and titanium }\end{array}$ \\
\hline Aurobindo Pharma Limited & $\begin{array}{l}\text { Confectioner's sug } \\
\text { magnesium stearat } \\
\text { cellulose, and talc. }\end{array}$ \\
\hline \multicolumn{2}{|l|}{ * Tested products } \\
\hline \multicolumn{2}{|l|}{ ACKNOWLEDGEMENTS } \\
\hline \multicolumn{2}{|c|}{$\begin{array}{l}\text { This research was supported by the University of } \\
\text { Alberta International, the Faculty of Pharmacy and } \\
\text { Pharmaceutical Sciences and DDIC. The authors like } \\
\text { to thank Dr. Casey Sayre for sourcing the USA } \\
\text { phenytoin formulations, Dr. Jack Cook for his } \\
\text { valuable comments on the study and Dr. Andrew J. } \\
\text { McLachlan for informing NMD of the initial } \\
\text { historical outbreak of phenytoin toxicity in Australia } \\
\text { decades ago. }\end{array}$} \\
\hline
\end{tabular}

Lactose, magnesium stearate, sugar and talc.

Lactose, magnesium stearate, sugar and talc.

\section{USA}


p. $13-23$

4. Hotha KK, Roychowdhury S, Subramanian V. Drug-Excipient Interactions: Case Studies and Overview of Drug Degradation Pathways. Am J Anal Chem. 2016;7(1):107-40.

5. Dilantin ${ }^{\circledR}$ [package insert]. Parke-Davis, Division of Pfizer, Inc., New York, NY; 2009.

6. Neuvonen PJ. Bioavailability of Phenytoin: Clinical Pharmacokinetic and Therapeutic Implications. Clin Pharmacokinet. 1979;4(2):91103.

7. Tyrer JH, Eadie MJ, Sutherland JM, Hooper WD. Outbreak of anticonvulsant intoxication in an Australian city. Br Med J. 1970;4(5730):271-3.

8. Bochner F, Hooper W, Tyrer J, Eadie M. The explanation of the 1968 Australian outbreak of diphenylhydantoin intoxication. Proc Aust Assoc Neurol. 1973;9:165-70.

9. Bochner F, Hooper WD, Tyrer JH, Eadie MJ. Factors Involved in an Outbreak of Phenytoin Intoxication. J Neurol Sci. 1972;16(4):481-7.

10. USP, UNITED States Pharmacopeia. 38. ed. Rockville: United States Pharmacopeial Convention, 2015.

11. Shah VP, Tsong Y, Sathe P, Liu JP. In vitro dissolution profile comparison- Statistics and analysis of the similarity factor, f2. Pharm Res. 1998;15(6):889-96.

12. Shah VP, Tsong Y, Sathe P, Williams RL. Dissolution Profile Comparison Using Similarit Factor , f2. Food and Drug Administration, Rockville, MD. 2017.

13. S. M. B, M. J. G. Some factors influencing the in vitro release of phenytoin from formulations. Int $\mathrm{J}$ Pharm. 1978;1(3):151-64.

14. Soryal I, Richens A. Bioavailability and dissolution of proprietary and generic formulations of phenytoin. J Neurol Neurosurg Psychiatry. 1992;55(8):688-91.

15. Pharmacopeial Forum - Online 41(6). Available from: http://www.usppf.com/pf/pub/index.html.

16. DW N. Phenyton injections: From compounding to Cerebyx. Int J Pharm Compd. 2002;6(6):4103.

17. Besag FMC. Is generic prescribing acceptable in epilepsy? Drug Saf. 2000;23(3):173-82.

18. Richens A. Clinical Pharmacokinetics of Phenytoin. Clin Pharmacokinet. 1979;4(3):15369.

19. LeLorier J, Duh MS, Paradis PE, Lefebvre P, Weiner J, Manjunath $\mathrm{R}$, et al. Clinical consequences of generic substitution of lamotrigine for patients with epilepsy. Health Care (Don Mills). 2008;2179-86.

20. Borgherini $\mathrm{G}$. The bioequivalence and therapeutic efficacy of generic versus brand-name psychoactive drugs. Clin Ther. 2003;25(6):157892.
21. Chowdhury DK, Sarker H, Schwartz P. Regulatory Notes on Impact of Excipients on Drug Products and the Maillard Reaction. AAPS PharmSciTech. 2017;19(2):965-9.

22. Bharate SS, Bharate SB, Bajaj AN. Interactions and incompatibilities of pharmaceutical excipients with active pharmaceutical ingredients : a comprehensive review. J excipients food Chem. 2010;1(3):3-26.

23. Stulova I, Kabanova N, Krisciunaite T, Adamberg K, Laht TM, Vilu R. Microcalorimetric study of the growth of Streptococcus thermophilus in renneted milk. Front Microbiol. 2015;6:3389.

24. Berridge NJ, Cousins CM, Cliffe AJ. Microcalorimetry applied to certain species of bacteria growing in sterilized separated milk. J Dairy Res. 1974;41(2):203-15.

25. Vickery RD, Maurin MB. Utility of microcalorimetry in the characterization of the browning reaction. J Pharm Biomed Anal. 1999;20(1-2):385-8.

26. Cavatur R, Murti Vemuri N, Chrzan Z. Use of isothermal microcalorimetry in pharmaceutical preformulation studies part III. Evaluation of excipient compatibility of a new chemical entity. J Therm Anal Calorim. 2004;78(1):63-72.

27. Choma CT. Characterization of Drug-Excipient Compatibility _ TA Instruments, 109 Lukens Drive, New Castle, DE 19720, USA.

28. Macheras P, Ismailos G, Reppas C. Bioavialability study of a freeze-dried sodium phenytoin-milk formulation. Biopharm Drug Dispos. 1991;12:687-95.

29. Neuvonen P, Lehtovaara R, Bardy A. Effect of Some Gastrointestinal Factors on Serum Phenytoin Concentration. Antiepileptic Ther Adv Drug Monit. 1980;149-59.

30. Dilantin ${ }^{\circledR}$ [Product monograph]. Pfizer Canada Inc., Licensee Kirkland, Québec; 2017.

31. Extended phenytoin sodium capsules, USP [Product monograph]. Taro Pharmaceuticals Inc., Brampton, Ontario; 2017.

32. Extended phenytoin sodium capsules, USP [Product monograph]. Apotex Inc., Toronto, Ontario; 2017.

33. USP, UNITED States Pharmacopeia. 40. ed. Rockville: United States Pharmacopeial Convention, 2017.

34. Cook J, Randinitis E, Wilder BJ. Effect of food on the bioavailability of 100-mg Dilantin Kapseals. Neurology. 2001;57:698-700.

35. Health Canada, Canada Vigilance program http://www.healthcanada.gc.ca/medeffect. Query number CV-2018-0060.

36. Extended phenytoin sodium capsules, USP [package insert]. Sun Pharmaceutical Industries, Inc., Cranbury, NJ; 2015.

37. Extended phenytoin sodium capsules, USP 
[package insert]. Amneal Pharmaceuticals LLC., New York, NY; 2017.

38. Extended phenytoin sodium capsules, USP [package insert]. Aurobindo Pharma USA, Inc.,North Dayton, NJ; 2015.

39. Extended phenytoin sodium capsules, USP [package insert]. Mylan Pharmaceuticals Inc.,Morgantown, WV; 2015.
40. Extended phenytoin sodium capsules, USP [package insert]. Taro Pharmaceuticals U.S.A., Inc., Hawthorne, NY; 2017.

41. Wilder BJ, Leppik I, Hietpas TJ, Cloyd JC, Randinitis EJ, Cook J. Effect of food on absorption of Dilantin Kapseals and Mylan extended phenytoin sodium capsules. Neurology. 2001;57:582-9. 